\title{
Reversible Intercalation of Fluoride-Anion Receptor Complexes in Graphite
}

\author{
William C. West, ${ }^{\mathrm{a}, *, \mathrm{z}}$ Jay F. Whitacre, ${ }^{\mathrm{a}, *}$ Nicole Leifer, ${ }^{\mathrm{b}}$ Steve Greenbaum, ${ }^{\mathrm{b}, *}$ \\ Marshall Smart, ${ }^{\mathrm{a}, *}$ Ratnakumar Bugga ${ }^{\mathrm{a}, *}$ Mario Blanco, ${ }^{\mathrm{c}}$ and \\ S. R. Narayanan ${ }^{\mathrm{a}, *}$
}

${ }^{a}$ Jet Propulsion Laboratory, California Institute of Technology, Pasadena, California 91109, USA

${ }^{b}$ Hunter College, City University of New York, New York, New York 10021, USA

${ }^{c}$ California Institute of Technology, Pasadena, California 91109, USA

\begin{abstract}
We have demonstrated a route to reversibly intercalate fluoride-anion receptor complexes in graphite via a nonaqueous electrochemical process. This approach may find application for a rechargeable lithium-fluoride dual-ion intercalating battery with high specific energy. The cell chemistry presented here uses graphite cathodes with LiF dissolved in a nonaqueous solvent through the aid of anion receptors. Cells have been demonstrated with reversible cathode specific capacity of approximately $80 \mathrm{mAh} / \mathrm{g}$ at discharge plateaus of upward of $4.8 \mathrm{~V}$, with graphite staging of the intercalant observed via in situ synchrotron X-ray diffraction during charging. Electrochemical impedance spectroscopy and ${ }^{11} \mathrm{~B}$ nuclear magnetic resonance studies suggest that cointercalation of the anion receptor with the fluoride occurs during charging, which likely limits the cathode specific capacity. The anion receptor type dictates the extent of graphite fluorination, and must be further optimized to realize high theoretical fluorination levels. To find these optimal anion receptors, we have designed an ab initio calculations-based scheme aimed at identifying receptors with favorable fluoride binding and release properties.
\end{abstract}

(C) 2007 The Electrochemical Society. [DOI: 10.1149/1.2759841] All rights reserved.

Manuscript submitted February 22, 2007; revised manuscript received May 24, 2007. Available electronically August 2, 2007.

As cathodes in lithium batteries, carbon-fluoride (C-F) compounds offer very high theoretical specific capacity, on the scale of $1785 \mathrm{mAh} / \mathrm{g}$ for $\mathrm{C}_{1.25} \mathrm{~F}$, though to date, all $\mathrm{C}-\mathrm{F}$ compounds regardless of preparation conditions are strictly non-rechargeable. A rechargeable $\mathrm{C}-\mathrm{F}$ cathode would be very desirable in terms of specific energy relative to conventional lithium-ion transition metal oxide cathodes, though there are numerous difficulties associated with reversible fluorination of carbon which relate to a large extent to the nature of the $\mathrm{C}-\mathrm{F}$ bond.

The reaction of carbon and fluorine is well known to yield a wide range of useful compounds with varying properties, depending on preparation conditions and synthesis route. Many of these compounds, despite their relatively poor electronic conductivity, are useful as cathodes for primary Li batteries. Direct reaction of fluorine gas with graphite at temperatures greater than $350^{\circ} \mathrm{C}$ results in covalent bonding of $\mathrm{C}_{x}-\mathrm{F}$ with the transition of the planar $\mathrm{sp}^{2}$ graphene sheets to buckled $\mathrm{sp}^{3}$ carbon, and concomitant drop in electrical conductivity with decreasing $x$. Room temperature chemical fluorination of graphite in the presence of various fluorides such as $\mathrm{HF}, \mathrm{WF}_{6}, \mathrm{SbF}_{5}$, and $\mathrm{IF}_{5}$ has been demonstrated by others up to $\mathrm{C}_{1.0} \mathrm{~F}_{0.89}\left(\mathrm{I}_{0.02} \mathrm{H}_{0.06}\right) .{ }^{1}$ Under these preparation conditions, the $\mathrm{sp}^{2}$ carbon hybridization is maintained, and the $\mathrm{C}-\mathrm{F}$ bonding is ionic. Electrochemical fluorination of graphite in aqueous or anhydrous $\mathrm{HF}$ media is also possible, resulting in ionic or semi-covalent $\mathrm{C}_{x}-\mathrm{F}$ depending on the degree of fluorination, though this process is not significantly reversible and has poor Coulombic efficiency. ${ }^{2-4} \mathrm{~A}$ candidate rechargeable $\mathrm{C}-\mathrm{F}$ cathode must retain good electronic conductivity and thus the practical lower bound on $\mathrm{C}_{x} \mathrm{~F}$ is likely near the $\mathrm{C}-\mathrm{F}$ delocalization composition at ca. $\mathrm{C}_{3.6} \mathrm{~F},{ }^{5}$ corresponding to a theoretical reversible cathode-specific capacity of $620 \mathrm{mAh} / \mathrm{g}$. The practical limit of fluorination can fall well below this theoretical specific capacity and still outperform state of charge lithium-ion battery cathodes, $\mathrm{LiCo}(\mathrm{Ni}) \mathrm{O}_{2}$, with cathode-specific capacity of about $180 \mathrm{mAh} / \mathrm{g}$.

The goal of this study was to examine the feasibility of preparing secondary batteries based on the C-F chemistry. The reversible fluorination of graphite using the principle of dual (de)intercalation of $\mathrm{Li}^{+}$at the anode and $\mathrm{F}^{-}$at the cathode opens the door to an interesting and potentially high-energy density variant on the Li-ion bat-

\footnotetext{
* Electrochemical Society Active Member.

${ }^{\mathrm{z}}$ E-mail: William.C.West@jpl.nasa.gov
}

tery cell design. Other groups ${ }^{6-9}$ have considered the advantages of a dual-ion intercalating battery, such as high cell voltage, using for example lithium cations and large polyatomic anions $\mathrm{PF}_{6}^{-}$or $\mathrm{ClO}_{4}^{-}$. These studies demonstrated successful cell operation, albeit with modest capacity and cycle life presumably due to the (de)intercalation of large polyatomic anions and poor anodic stability of the electrolyte solvent. One research group also identified a key limitation to practical application using this design: high-capacity cells require high electrolyte salt loading that results in poor electrolyte conductivity. ${ }^{10}$ To address these important issues, we have examined the possibility of using fluoride as an anion for intercalation into graphite cathodes for rechargeable lithium-ion batteries.

The cell chemistry presented here specifically addresses several of the key limitations of previous designs of dual-ion intercalating cell designs, using graphitic anodes and cathodes but employing $\mathrm{LiF}$ as the functional salt, dissolved in nonaqueous, high-voltage stability organic solvents through the aid of anion complexing additives. Unlike conventional $\mathrm{Li}$-ion cells, the salt in the electrolyte of a dual-ion intercalating cell is depleted from and returned to the electrolyte during cell charging and discharging, respectively. Typically, $1 \mathrm{M} \mathrm{Li}$ salt provides the optimal electrolyte resistance in Li-ion cells, with deviations above and below this molarity resulting in increases in electrolyte resistance. An important consequence of using anion receptors to solvate the $\mathrm{LiF}$ is that the salt concentration in the electrolyte can be kept near optimal values by using a $1 \mathrm{M}$ solution of monodentate receptor in the solvent. Additional salt can be added to the electrolyte or electrodes as a precipitate, functioning as a reservoir during charging. Assuming the salt precipitation and dissolution is not the cell rate-limiting step, as the anion receptor releases $\mathrm{F}^{-}$at the high anodic potentials at the cathode, the electrolyte resistance can be kept relatively constant despite the high salt loading in the cell.

The utility of fluorinated boron-based anion receptors for complexation of lithium halide salts in nonaqueous solvents has been reported by others. ${ }^{11}$ These materials have been observed to enhance the ionic disassociation of a variety of lithium salts in low dielectric solvents, by incorporating non-hydrogen-bonded electron withdrawing groups that are stable over a wide electrochemical stability window of at least $5 \mathrm{~V}^{12}$ These compounds, such as tris(pentafluorophenyl) borane (TPFPB), when dissolved in conventional Li-ion battery electrolyte solvents such as ethers and aliphatic carbonates enhance the dissolution of lithium salts, including lithium halides, resulting in increases in solubilities by upwards of six orders of 
magnitude. The conductivity of these electrolyte solutions is nearly on par with conventional Li-ion electrolyte solutions, e.g., 3.58 $\times 10^{-3} \mathrm{~S} / \mathrm{cm}$ for $1 \mathrm{M} \mathrm{LiF}$ in $1: 2$ ethylene carbonate-dimethyl carbonate (EC-DMC) for $1 \mathrm{M}$ anion receptor at $25^{\circ} \mathrm{C}^{13}$

\section{Experimental}

Cell fabrication.- Although the dual-ion intercalation cell design uses graphite for both electrodes, this study focused primarily on the cathode ( $\mathrm{C}-\mathrm{F}$ ) reactions because $\mathrm{Li}$ (de)intercalation into graphite anode is a well-understood mechanism. Test cells used for cathode specific capacity and cycling experiments were constructed using stainless-steel CR3032 coin cell hardware with Al cladding at the cathode terminal. The cells were designed to be cathode limited, with excess $\mathrm{Li}$ and $\mathrm{LiF}$ relative to the cathode. The anodes were stamped from Li foil, and the graphite (Alfa Aesar) cathodes were solvent cast onto $\mathrm{Al}$ foil from a mix of $80 \mathrm{wt} \%$ graphite, 10 wt $\%$ carbon black (Shawinigan), and $10 \mathrm{wt} \%$ polyvinylidene fluoride (PVDF) binder (Aldrich, $\mathrm{MW}_{\mathrm{avg}}=534,000$ ) in 1-methyl 2-pyrrolidone (Omnisolv), to a graphite loading of ca. $5 \mathrm{mg} / \mathrm{cm}^{2}$. The cathode foils were vacuum dried prior to cell assembly. The electrolyte consisted of a saturated solution of LiF, high anodic stability aprotic solvents such as ethyl isopropyl sulfone (EIS, TCI America) ${ }^{14}$ and propylene carbonate (PC, Mitsubishi), and ca. 1-2 $\mathrm{M}$ anion receptor. Some electrolyte formulations with high anion receptor molarities were slurries rather than single-phase solutions. Several anion receptor variants were examined, including tris(2, 2, 2 trifluoroethyl borate) (Richman Chemicals), and bis(1, 1, 1, 3, 3, 3-hexafluoroisopropyl) pentafluorophenylboronate (Richman Chemicals), though cell data represented here were collected from cells using either TPFPB (Aldrich) or tris(hexafluoroisopropyl) borate (THFIPB, TCI America and Richman Chemicals). To assure the cell operated in a cathode-limited manner, LiF (EM Industries) was added to ca. 10-20 times stoichiometric value assuming C:F of 1:1. This excess LiF cannot be dissolved in the electrolyte and instead was present as a solid suspended in the anion receptor-solvent solution. No other salt aside from LiF was added to the electrolyte. The two anion receptors were used as received without further purification. These anion receptors were critical for the cell operation given that $\mathrm{LiF}$ is insoluble in virtually all neat organic solvents.

Electrochemical characterization- - For cyclic voltammetry measurements for Li plating-stripping characterization, the coin cell was modified so that the graphite electrode was replaced with a bare coin cell current collector sputter-coated with ca. $100 \mathrm{~nm} \mathrm{Cu}$. Cyclic voltammetry measurements for anodic oxidative stability characterization were carried out using a glass cell with Li foil reference and counter electrodes and a Pt working electrode (Bioanalytical Systems).

The test cells characterized via electrochemical impedance spectroscopy (EIS) and cyclic voltammetry (CV) techniques were performed using a Princeton Applied Research VMP2 frequency response analyzer-potentiostat, and a Princeton Applied Research $273 \mathrm{~A}$ potentiostat. CV measurements were performed with sweep rates between 0.1 and $5 \mathrm{mV} / \mathrm{s}$. The applied ac signal for the EIS measurements was $20 \mathrm{mV}$, with frequencies between $100 \mathrm{kHz}$ and $0.1 \mathrm{~Hz}$.

Synchrotron X-ray diffraction.- In situ X-ray diffraction cell characterization was performed at the Stanford Synchrotron Radiation Laboratory (SSRL). To maximize the scattered signal, the asymmetric GIXS geometry was used on Beamline 2-1 at SSRL $(8 \mathrm{keV})$. A silicon (111) monochromator was used to select the wavelength of the X-ray beam, $0.154996 \mathrm{~nm}$, in focused mode. The vertical and horizontal divergences of the beam were 0.2 and $3 \mathrm{mrad}$, respectively. Slits $1 \times 1 \mathrm{~mm}$ in area were used to define the incident beam and a second $\mathrm{Si}(111)$ monochromator was used on the detector arm. Diffracted X-ray intensity was measured using a standard scintillation counter. Samples were manipulated using an automated Hüber two-circle diffractometer that was controlled using a UNIX workstation. The incident beam dose was measured by a scintillation detector placed upstream, off of a beam-splitter, from the incident beam slits. All measurements were conducted using a set dose for each data point, eliminating any possible experimental error due to beam intensity fluctuation.

${ }^{11} \mathrm{~B}$ solid state NMR.— ${ }^{11} \mathrm{~B}$ solid-state nuclear magnetic resonance (NMR) experiments were conducted on an $11.7 \mathrm{~T}(1 \mathrm{H}$ resonance frequency: $500 \mathrm{MHz}$ ) wide-bore instrument (Varian) equipped with a $4 \mathrm{~mm}$ magic angle spinning (MAS) probe at 160.356 MHz. All experiments were carried out at room temperature. The MAS frequency was set to $10 \mathrm{kHz}$. The ${ }^{11} \mathrm{~B}$ resonances were calibrated using $1 \mathrm{M} \mathrm{NaBH}_{4}$ as an external reference at $-38.7 \mathrm{ppm}^{15}$ Two cells were charged at a constant current of $0.500 \mathrm{~A}$ until reaching a voltage of $5.35 \mathrm{~V}$; both cells were then held at this voltage for $1 \mathrm{~h}$. One cell was discharged at constant current of $0.500 \mathrm{~A}$, held at $3.0 \mathrm{~V}$, and was then disassembled inside an Ar glove box. Half of the cathode material from each cell was subject to a rinsing procedure in which the substances were subject to three separate 10 min soaks in previously dried dimethyl carbonate (DMC). The cells were then packed into $4 \mathrm{~mm}$ NMR rotors.

\section{Results and Discussion}

Electrochemical characterization.- Figure 1a and b shows representative slow charge-discharge characteristics of graphite cathode cells, fabricated using electrolytes consisting of propylene carbonate (PC), LiF, and TPFPB and THFIPB anion receptors, respectively. The discharge capacity was typically about $60 \mathrm{mAh} / \mathrm{g}$ above $2 \mathrm{~V}$ when using the TPFPB anion receptor and $80 \mathrm{mAh} / \mathrm{g}$ using THFIPB anion receptor. Although PC is well known to co-intercalate with $\mathrm{Li}^{+}$ in graphite at low $\mathrm{Li} / \mathrm{Li}^{+}$potentials near $1.5 \mathrm{~V}$, resulting in massive irreversible capacity and destruction of the graphite via exfoliation in one or two cycles, we observe that at high $\mathrm{Li} / \mathrm{Li}^{+}$potentials of ca. $3-5.5 \mathrm{~V}$, PC does not apparently co-intercalate; cells could be charged and discharged at least 90 cycles or more, with minimal capacity fade (Fig. 1c). Cells fabricated using THFIPB typically experienced a minor increase in cathode specific capacity over the first few cycles, followed by a more gradual decrease in specific capacity. Cells fabricated with TPFPB experienced a steep rise in cathode-specific capacity from near-zero capacity at the first few cycles to about $60 \mathrm{mAh} / \mathrm{g}$ specific capacity after roughly 100 cycles, followed by a more gradual decrease in specific capacity with cycling. The origin of the initially low and subsequent increasing specific capacity with cycling using TPFPB is not clear, though this may be due to poor wetting of the cathode because the TPFPB solutions were more viscous than THFIPB solutions.

Depending on anion receptor type, charge-discharge cutoff voltages, and current density, the overall Coulombic efficiency was about $75 \%$, well above other electrochemical fluorination methods, but below that which would be desirable for rechargeable batteries. High anodic stability solvents must be identified which can tolerate these high-charge voltages in order to limit the irreversible capacity losses. To this end, we performed CV measurements to examine the anodic stability of the two different anion receptors. Electrolytes with either anion receptor exhibited an onset of an anodic peak at roughly the same potential of about $5 \mathrm{~V}$ vs $\mathrm{Li} / \mathrm{Li}^{+}$, with a much stronger, well-defined peak observed for the TPFPB solution (Fig. 2a). However, in this cell configuration, we were unable to attribute the anodic current to anion receptor-fluoride decomplexation followed by oxidation of fluoride, or parasitic oxidation of the solvent or anion receptor. It is clear that the solvent-anion receptor system can tolerate high anodic potentials without appreciable oxidation to near the potentials where we would expect the onset of fluoride intercalation.

In addition to appropriate anodic stability, the electrolyte solutions must be chemically stable or form stable passivation films at the anode. To probe the reductive stability of the electrolyte solutions, a $\mathrm{Cu}$ working electrode was driven to negative potentials vs $\mathrm{Li} / \mathrm{Li}^{+}$, and then the potential sweep was reversed to induce positive 

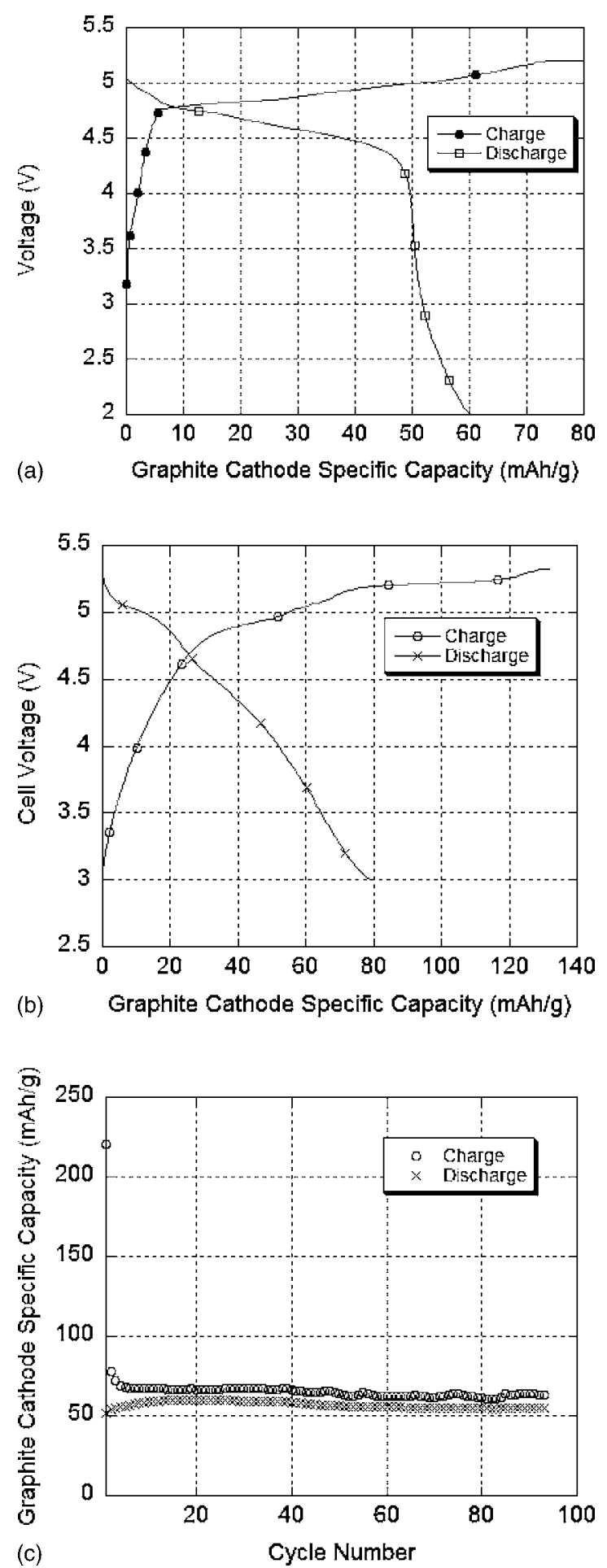

Figure 1. Charge-discharge plot of graphite cathode specific capacity as a function of cell voltage, with the cell fabricated using $0.95 \mathrm{M}$ TPFPB, excess $\mathrm{LiF}$ (ca. $20 \mathrm{M}$ ) in PC at current density of $0.28 \mathrm{~mA} / \mathrm{cm}^{2}$ and electrode loading of $5.1 \mathrm{mg} / \mathrm{cm}^{2}$ (a), and $1.8 \mathrm{M}$ THFIPB, excess (ca. $20 \mathrm{M}$ ) in PC at current density of $0.056 \mathrm{~mA} / \mathrm{cm}^{2}$ and electrode loading $4.5 \mathrm{mg} / \mathrm{cm}^{2}(\mathrm{~b})$, and cathode-basis specific capacity as a function of cycle number for a cell fabricated using $1.8 \mathrm{M}$ THFIPB, excess (ca. $20 \mathrm{M}$ ) in PC, at higher current density of $0.28 \mathrm{~mA} / \mathrm{cm}^{2}$, electrode loading of $5.5 \mathrm{mg} / \mathrm{cm}^{2}$ (c).

potentials. Provided the electrolyte solution is reductively stable to $\mathrm{Li}$, then $\mathrm{Li}$ metal should be stripped from the $\mathrm{Li}$ counter electrode and plated on the $\mathrm{Cu}$ working electrode when the potential of the working electrode falls below $0 \mathrm{~V}$ vs $\mathrm{Li} / \mathrm{Li}^{+}$. As the voltage sweep
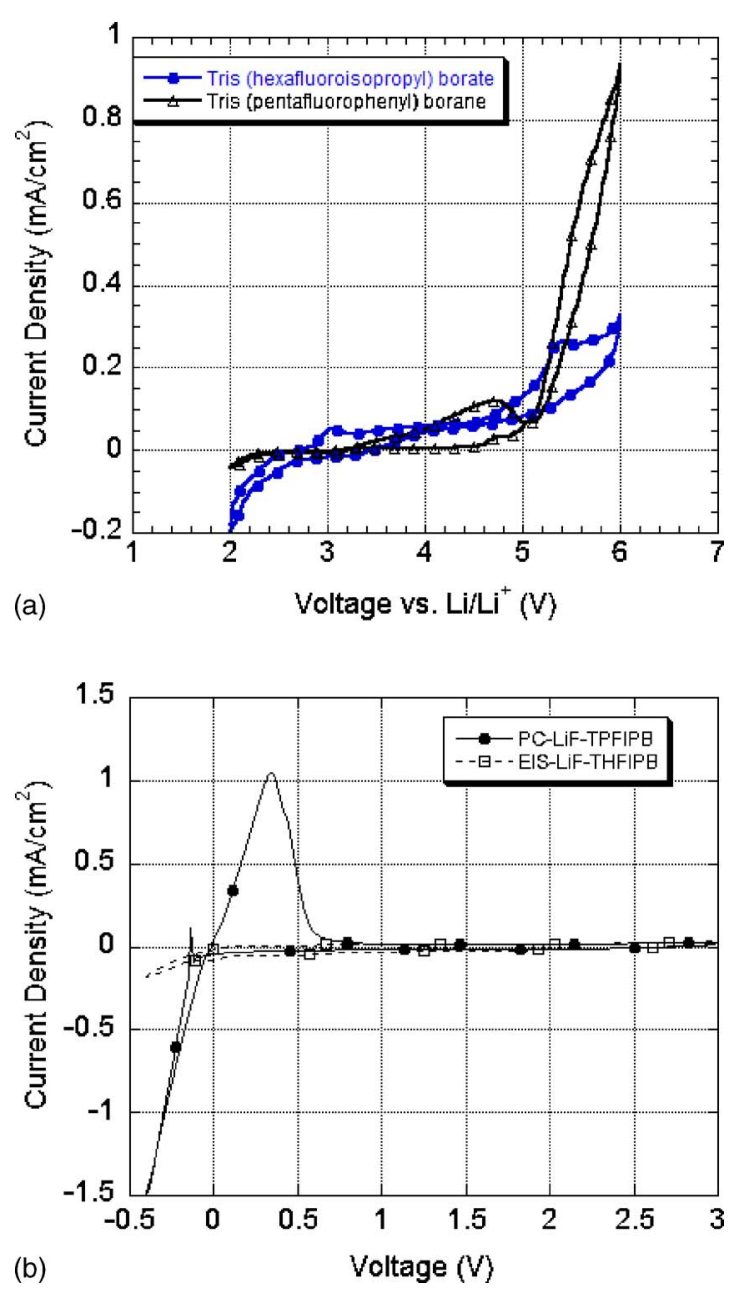

Figure 2. (Color online) (a) CV measurements of two electrolyte solutions (Pt working electrode, Li foil counter and reference electrodes). The TPFPB electrolyte solution was $0.26 \mathrm{M}$ TPFPB, $0.36 \mathrm{M} \mathrm{LiF}$ in PC, and the THFIPB solution was $0.26 \mathrm{M}$ THFIPB, $0.36 \mathrm{M} \mathrm{LiF}$ in PC. (b) The reductive stability against a Li anode was studied for two electrolytes with $0.48 \mathrm{M}$ THFIPB, excess LiF in PC and 0.55 M THFIPB, excess LiF in ethyl isopropyl sulfone (EIS). CV scans ( $\mathrm{Cu}$ working electrode and $\mathrm{Li}$ foil counter electrode) demonstrated that plating and stripping of $\mathrm{Li}$ onto $\mathrm{Cu}$ was possible only for $\mathrm{PC}$ and not for EIS electrolyte solutions.

is reversed and the potential at the working electrode rises above $0 \mathrm{~V}$ vs $\mathrm{Li} / \mathrm{Li}^{+}$, any plated $\mathrm{Li}$ on the $\mathrm{Cu}$ working electrode should be stripped and replated at the Li counter. If, however, the electrolyte is not stable in contact with Li metal, then the small amount of plated $\mathrm{Li}$ metal at the $\mathrm{Cu}$ working electrode will react with the electrolyte and will not be stripped from the $\mathrm{Cu}$ working electrode. In this manner, it is possible to probe the relative stability of the electrolytes to $\mathrm{Li}$ metal.

For the LiF-PC-THFIPB solution we observed a sharp negative spike in current as the voltage becomes negative relative to the $\mathrm{Li}$ counter, while the cell with LiF-EIS-THFIPB solution exhibits a much weaker rise in current as the potential crosses $0 \mathrm{~V} \mathrm{Li} / \mathrm{Li}^{+}$and becomes more negative (Fig. 2b). When the potential sweep is reversed and the $\mathrm{Cu}$ working electrode is polarized positive relative to the Li counter electrode, we observed a well-defined anodic current peak attributed to $\mathrm{Li}$ stripping at the $\mathrm{Cu}$ working electrode for the LiF-PC-THFIPB solution, but no positive anodic current for the LiF-EIS-THFIPB solution. We conclude that the PC-based electrolytes, when coupled with THFIPB, had far superior reductive stability against Li plating and stripping in comparison to the sulfonebased electrolytes. While the PC-based electrolytes have excellent 

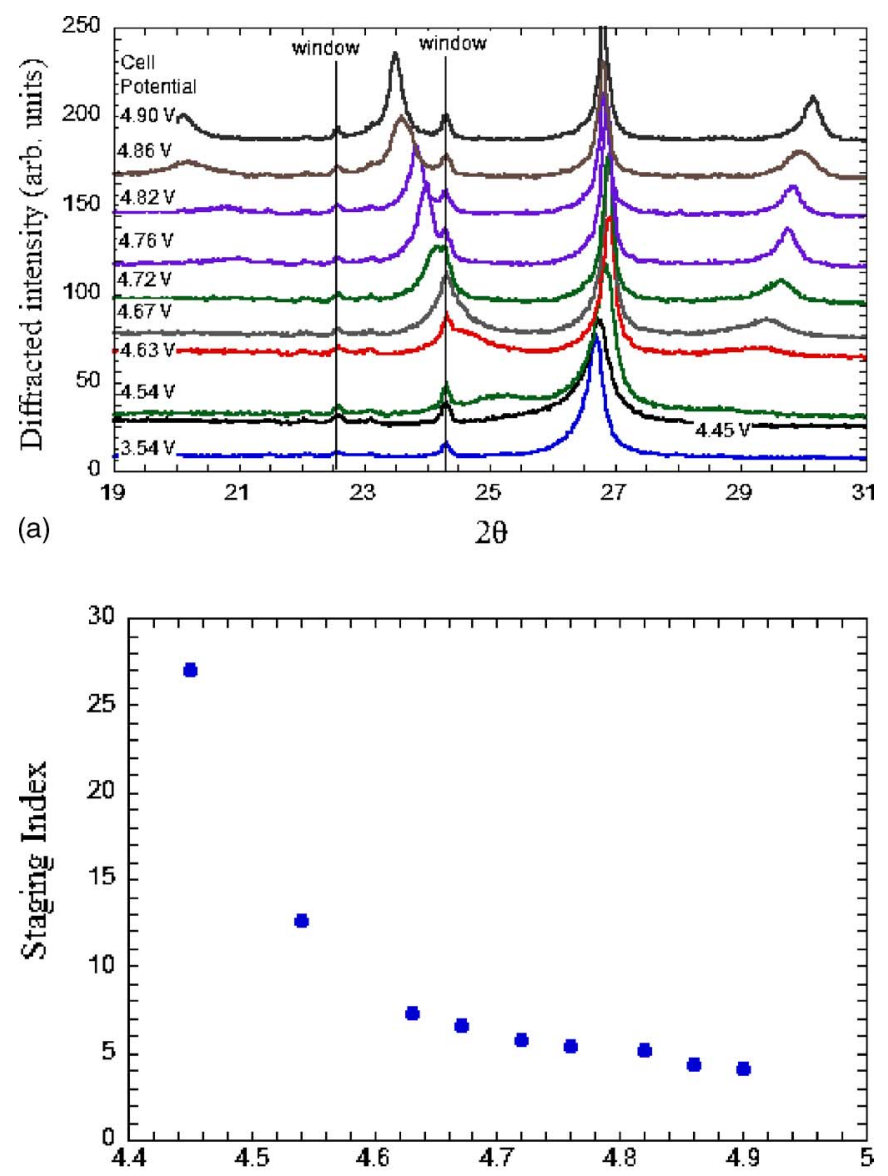

(b)

\section{Cell Potential (v)}

Figure 3. (Color online) Synchrotron X-ray diffraction data of a graphite cathode as function of state of charge, with the $\mathrm{Al}$ cell window demarcated (a), and staging index as a function of cell potential. The electrolyte was 1.2 M THFIPB in excess LiF (ca. 20 M) in PC. At a low state of charge (3.54 $\mathrm{V}$ and lower), only the main graphitic (002) diffraction peak was evident. Upon charging, the (002) peak moved to a higher $2 \theta$ value, while new maxima evolved, and moved about $2 \theta$ values of $25^{\circ}(4.54 \mathrm{~V}), 29.5^{\circ}$ $(4.67 \mathrm{~V})$, and eventually $20^{\circ}(4.86 \mathrm{~V})$. This pattern of peak evolution and movement as a function state of charge is consistent with the occurrence of gallery staging.

reductive stability against $\mathrm{Li}$ anodes, these electrolyte co-intercalate with $\mathrm{Li}$ in graphitic anodes. As such, alternative solvents are currently being sought that are compatible with graphitic anodes.

Synchrotron X-ray diffraction.- The structural behavior of the graphitic cathode material was examined in situ during electrochemical cycling using synchrotron X-ray diffraction. Figure $3 \mathrm{a}$ shows the diffraction data plotted as a function of state of charge. At a low state of charge ( $3.54 \mathrm{~V}$ and lower), only the main graphitic (002) diffraction peak was evident. Upon charging, the (002) peak moved to a slightly higher $2 \theta$ value, consistent with the formation of a dilute stage $1 \mathrm{C}-\mathrm{F}$ compound with smaller $d_{(002)}$ spacing than pristine graphite. With increasing charge voltage, new maxima evolved, and shifted about $2 \theta$ values of $25^{\circ}(4.54 \mathrm{~V}), 29.5^{\circ}$ $(4.67 \mathrm{~V})$, and eventually $20^{\circ}(4.86 \mathrm{~V})$. No peaks evolved below $2 \theta$ values of $19^{\circ}$. This pattern of peak evolution and movement as a function of state of charge (or other chemical-electrochemical influence) is consistent with the occurrence of gallery staging of intercalants in graphite. The effect was fully reversible upon cell discharge and was observed in multiple test cells. Figure $3 \mathrm{~b}$ shows a plot of calculated staging index as a function of state of charge. The
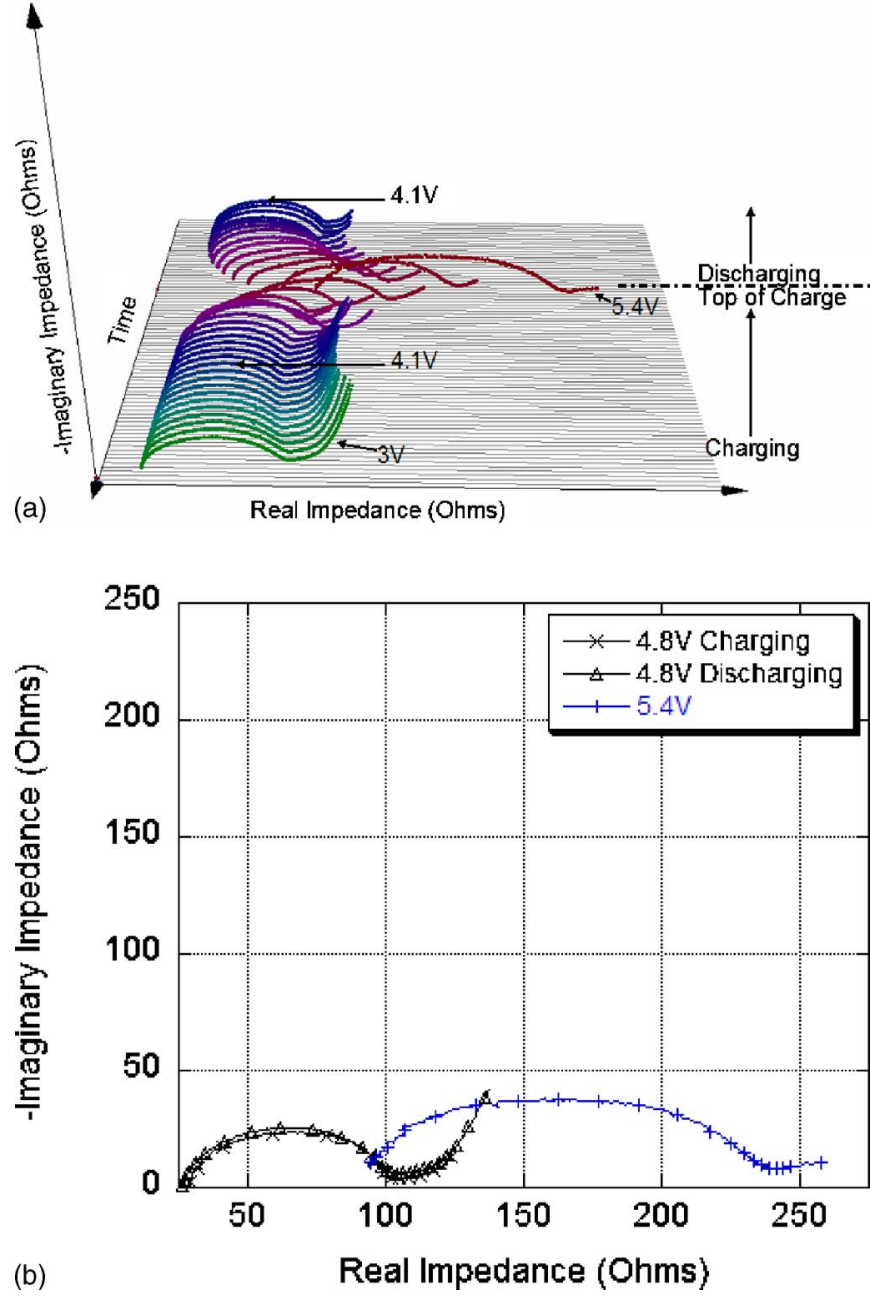

Figure 4. (Color online) EIS data as a function of state of charge, across the entire charge-discharge range (a) and at two different cell voltages (b). The electrolyte was $0.71 \mathrm{M}$ THFIPB in excess LiF (ca. $20 \mathrm{M}$ ) in PC. The cell impedance increases with cell voltage at the high-frequency real impedance axis intercept which is associated with electrolyte conductivity. The impedance increases and decreases are consistent salt uptake and rejection with charging and discharging.

intercalate gallery height was calculated to be $9.90 \pm 0.05 \AA$ above $4.76 \mathrm{~V}$, where the bulk of the electrochemical capacity was observed.

This gallery height is greater than that expected for ionically bound fluoride in graphite $(2.7 \AA),{ }^{16}$ though smaller than that observed for the intercalation of large triflate imide and fluoroborate anions at about $13-15 \AA .{ }^{17}$ These results suggest that the reversible co-intercalation of the tightly bound anion receptor-fluoride complex occurred during cycling.

EIS. - To further assess the hypothesis of co-intercalation of the anion receptor with the fluoride on charging, we carried out EIS measurements as a function of state of charge. As the cell charged and the $\mathrm{Li}^{+}$and $\mathrm{F}^{-}$ions intercalated into the anode and cathode, respectively, the cell impedance increased, in particular at the highfrequency real impedance axis intercept associated with electrolyte conductivity (Fig. 4a). This increase in cell impedance was fully reversible on discharge (Fig. 4b). In principle, the cell impedance should not increase appreciably during charging because an excess of $\mathrm{LiF}$ is present in the cell, unless dissolution of the salt is the rate limiting process. However, we found that this impedance increase with charging was qualitatively time invariant, in that the cell impedance did not change with time as the cell at a constant high 


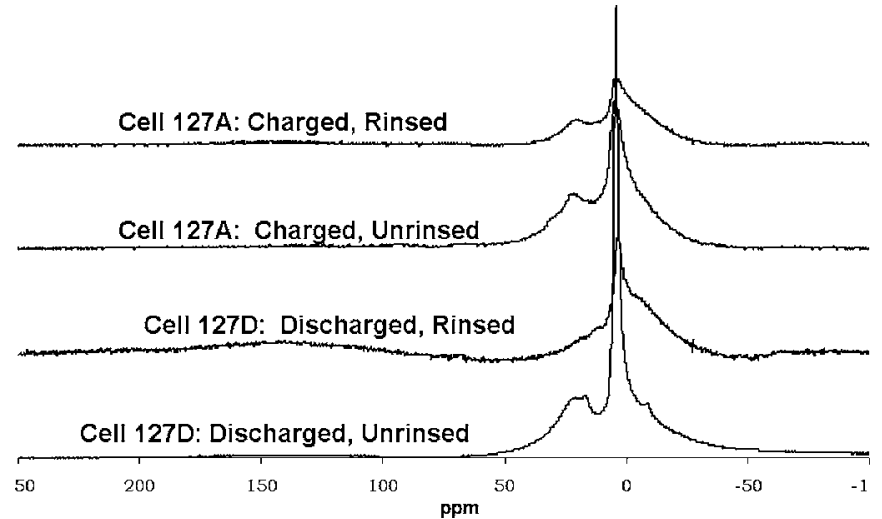

Figure 5. ${ }^{11}$ B MAS NMR of two cells, pre- and postrinse at the charged and discharged states. The electrolyte was 2.5 M THFIPB, excess LiF (ca. $20 \mathrm{M}$ ) in PC. In all but the rinsed, discharged sample (127D _ rinsed) a broad peak is seen at approximately $18 \mathrm{ppm}$, which corresponds to the chemical shift of the anion receptor salt, suggesting at least partial co-intercalation of the anion receptor.

charge voltage. Because the degree of fluorination at the top of charge was comparatively low, on the order of about $\mathrm{C}_{44} \mathrm{~F}$, little increase in the graphite cathode electronic conductivity with fluorination was expected. Thus, it is very likely the anion receptor does not fully release $\mathrm{F}^{-}$during charge, and accompanies the anion into the cathode, resulting in a decrease in electrolyte molarity and commensurate increase in electrolyte impedance. Nonetheless, the impedance of the cell was observed to be quite reversible with charging and discharging, suggesting that the anion receptor does not remain in the graphite cathode following discharge.

${ }^{11} B$ NMR. $-{ }^{11} \mathrm{~B}$ NMR data further supported the above claim that at least to some extent co-intercalation of the anion receptor with the $\mathrm{F}^{-}$occurs, which may explain the limited cathode-specific capacity on these initial cells because the intercalated bulky anion receptor molecule will sterically hinder $\mathrm{F}^{-}$intercalation. The four NMR spectra shown in Fig. 5 were digitally scaled equally; however, the mass of each sample could not be accurately measured and therefore the relative scales of the spectra were not normalized to the sample mass. In all four spectra an unidentified sharp peak was observed at approximately $3 \mathrm{ppm}$. In all but the rinsed, discharged sample, a broad peak was observed at approximately $18 \mathrm{ppm}$, which corresponds to the chemical shift of the anion receptor. The difference in the widths of the peaks was attributed to significantly smaller nuclear quadrupole broadening of the $3 \mathrm{ppm}$ peak. There also appeared to be an unidentified broad peak shifted upfield, at approximately $130 \mathrm{ppm}$, which we speculate to be associated with the intercalated receptor. The appearance of the anion receptor chemical shift for the charged and rinsed sample strongly suggested that co-intercalation of the anion receptor in the graphite occurs during charging. In both cells, this component was seen more clearly in the rinsed versions because the signal was not saturated by the stronger, surface anion receptor signal. The small chemical shift, lack of quadrupole broadening, and long spin-lattice relaxation time of the narrow peak near $3 \mathrm{ppm}$ imply that it corresponds to boron in a highly symmetric environment in a semisolid breakdown component of the anion receptor. Although preliminary ${ }^{19} \mathrm{~F}$ NMR studies were also carried out to determine the degree of anion receptor and $\mathrm{F}^{-}$co-intercalation, these studies were hindered by the large $\mathrm{F}$ background signal associated with the fluorinated binder and anion receptor.

Ab initio modeling.- Although a number of different variables (e.g., electrolyte composition, electrode loadings, and carbon types) were explored, we have found that the overarching variable dictating cell performance in terms of charge-discharge behavior was the an- ion receptor chemical compound type; all receptors were associated with unique charge-discharge voltage profiles and maximum specific capacity. For the two representative anion receptors presented here, we found that the highest performance anion receptor in terms of cathode-specific capacity, THFIPB, also co-intercalated into the cathode, which worsens the cathode-specific capacity. In order to obtain the maximal specific capacity, it is necessary to identify anion receptors and associated solvents with the following optimal characteristics:

1. Sufficiently wide electrochemical stability window, viz., will not anodically oxidize at the cathode during charging, and will not cathodically reduce at the anode.

2. Moderately strong fluoride affinity: If the anion receptor affinity for $\mathrm{F}^{-}$is too weak, the LiF will not be drawn into solution at sufficient molarity. If the fluoride affinity is too strong, the anion receptor will not release the bound $\mathrm{F}^{-}$during charging, and will co-intercalate into the graphite cathode.

3. High solubility and diffusivity of the anion receptor complex in the electrolyte.

Critical to cell operation is the release of the anion from the receptor at the cathode/electrolyte interface during charging. To this end, the binding energy of the anion complexing agent can be tailored by manipulating the chemistry of the complexing agents; e.g., electron deficient $\mathrm{N}$ or B atoms or electron withdrawing groups can be substituted with weaker binding atoms or groups of atoms. We have developed a first principles calculations-based scheme to identify optimal anion binding energetics, able to both draw sufficient $\mathrm{LiF}$ into the solvent as well as releasing the anion at charge potentials near $5 \mathrm{~V}$ vs $\mathrm{Li} / \mathrm{Li}^{+}$. To this end, we investigated the structures, solvent effects, and binding energetics of the two anion receptors using density functional theory (DFT) quantum mechanics.

Due in part to its wide use, and our previous experience with similar systems, ${ }^{18}$ we employed the Becke-Lee-Yang-Parr 3parameter (B3LYP) functional ${ }^{19}$ in the Jaguar quantum suite of programs. ${ }^{20}$ All anion acceptor geometries, in their neutral and charged states, were optimized in vacuum using the LACVP (Ref. 21) basis set with added double polarization and diffuse functions, LACVP**++ . A typical calculation contains over 600 basis functions.

We conducted geometry optimization, starting with the B3LYP/LACVP ${ }^{* *++}$ vacuum optimized structures, inside a continuum dielectric medium with a dielectric constant $\varepsilon=64.4$ which corresponds to propylene carbonate (PC). In this approach we obtained minimum-energy solvated structures with a self-consistent reaction field method, using a Poisson-Boltzmann solver. ${ }^{19,22}$ Solvation energies from the geometry optimizations were computed as the difference between the energy of the optimized gas phase structure and the energy of the solvated structure that was optimized in solution. In this method, after the usual gas phase wave function was calculated, we calculated the electrostatic potential, and fitted that potential to a set of atomic charges. These charges were passed to the Poisson-Boltzmann solver, which then determined the reaction field by numerical solution of the Poisson-Boltzmann equations, which then represented the solvent as a layer of charges at the molecular surface, the dielectric continuum boundary. The molecular surface was obtained by assigning a probe radius of $2.7 \AA$ to PC, a value that reproduced its liquid density. These solvent point charges were returned to Jaguar's SCF program, which performed another quantum-mechanical wave function calculation incorporating the solvent charges. This process was repeated until self-consistency was obtained. We have used a similar method to estimate complexation constants for yttrium-90 in DOTA. ${ }^{23}$ To aid the understanding of the anion complexation process we first reviewed the energetics of $\mathrm{LiF}$.

The experimental atomization energy of LiF crystal is 137.7 and $138.6 \mathrm{kcal} / \mathrm{mol}( \pm 2 \mathrm{kcal} / \mathrm{mol})$ at 0 and $298.15 \mathrm{~K}$, respectively. ${ }^{24}$ The ionization energy and electron affinity of $\mathrm{Li}$ and $\mathrm{F}$ are 5.39 and 


\begin{tabular}{|c|c|c|c|c|c|c|c|}
\hline ТРFPB & Receptor & Receptor $+\mathrm{F}^{-}$ & & THFIPB & Receptor & Receptor + F- & \\
\hline Symmetry & $\mathrm{C} 3$ & $\mathrm{C} 3$ & $q$ transfer & & $\mathrm{C} 3 \mathrm{~h}$ & $\mathrm{C} 3$ & $q$ transfer \\
\hline Dipole $\mu$ & 0.27 & 2.45 & & & 0 & 2.94 & \\
\hline$q(\mathbf{B})$ & 0.32 & 0.41 & 0.09 & & 0.65 & 1.51 & 0.86 \\
\hline$q(\mathrm{~F})$ & -0.27 & -0.29 & -0.3 & & -0.28 & -0.31 & -0.54 \\
\hline$q(\mathrm{~F}-)$ & & -0.47 & -0.53 & & & -0.62 & -0.38 \\
\hline$q(\mathrm{C}: \mathrm{B})$ & -0.14 & -0.11 & 0.09 & $q(\mathrm{O}: \mathrm{B})$ & -0.486 & -0.66 & -0.522 \\
\hline \multirow{3}{*}{$q(\mathrm{C})$} & 0.28 & 0.25 & -0.45 & $q(\mathbf{C})$ & 0.83 & 0.93 & 0.6 \\
\hline & & & & $q(\mathrm{C}: \mathrm{O})$ & 0.06 & -0.15 & -0.72 \\
\hline & & & & $q(\mathbf{H})$ & 0.21 & 0.19 & -0.02 \\
\hline$R(\mathbf{F E F})$ & 4.55 & 4.67 & & & 4.24 & 5.52 & \\
\hline$R(\mathbf{B}-\mathrm{C})$ & 1.57 & 1.66 & & R(B-O) & 1.366 & 1.47 & \\
\hline$R($ FEF-) & & 2.72 & & & & 3.19 & \\
\hline$R(\mathrm{~B}-\mathrm{F}-)$ & & 1.44 & & & & 1.43 & \\
\hline
\end{tabular}

$-3.40 \mathrm{eV},{ }^{25,26}$ respectively (the values calculated in this work were 5.62 and $-3.54 \mathrm{eV}$, respectively). The solvation of $\mathrm{Li}^{+}$and $\mathrm{F}^{-}$in $\mathrm{PC}$ were calculated to be -135.16 and $-94.36 \mathrm{kcal} / \mathrm{mol}$. Thus, the creation of a solvated $\mathrm{Li}^{+} \ldots \mathrm{F}^{-}$pair in pure $\mathrm{PC}$ from the crystal yielded an enthalpy of $-45.9 \mathrm{kcal} / \mathrm{mol}$. Surface effects, which were ignored here, make the actual enthalpy slightly more favorable. One might conclude that $\mathrm{LiF}$ is infinitely soluble in PC. However, the solvation energies calculated here are for infinite dilution in PC solvent. As soon as a small amount of LiF goes into solution, the capacity of PC to dissolve more $\mathrm{LiF}$ is diminished.

Table I presents the calculated structural data for the two anion receptors in their neutral and charged forms. Upon binding to $\mathrm{F}^{-}$, TPFPB keeps its C3 symmetry, but the dipole moment, calculated from the wave function, increases significantly (from $\mu=0.27$ to $\mu=2.45$ debye). Charge transfer from the $\mathrm{F}^{-}$anion to the receptor has been calculated as the difference between the Mulliken charges before and after anion capture. For example, $q\left(\mathrm{~F}^{-}\right)$is -0.47 electrons after capture, thus representing a net loss of -0.53 e from the free $\mathrm{F}^{-}$anion state $(-1 \mathrm{e})$. Fluorine atoms that form part of the TPFPB anion receptor suffer small changes in charge as a result of binding. However, because there are 15 such fluorine atoms, their combined charge transfer is significant, -0.3 .

In TPFPB the phenyl rings accommodate the anion charge. Total charge transfer from the $\mathrm{F}^{-}$to the receptor is larger in TPFPB than in THFIPB, -0.53 vs -0.38 electrons. This explains a higher anion binding energy for TPFPB as shown in Table II. In THFIPB, the oxygen, hydrogen, and fluoride atoms are all responsible for receiving the $\mathrm{F}^{-}$charge. However, the overall carbon charge transfer contribution is positive ( +0.3 electrons).

For the unbound THFIPB receptor the three remaining hydrogen atoms are all on the same plane as the borate group (Fig. 6c), giving a high $\mathrm{C} 3 \mathrm{~h}$ symmetry to the unbound receptor and a zero dipole moment. Upon binding, the hydrogens reorient out of the plane (Fig. $6 \mathrm{~d})$, lowering the symmetry to $\mathrm{C} 3$. The oxygen atoms and the carbon atoms bound to them (labeled C:O in Table I) in THFIPB are responsible for accepting roughly half the anion charge. Note that the calculated Mulliken charges are consistent with the large dipole moment showing a highly polarized $\mathrm{B} \ldots \mathrm{F}^{-}$bond in THFIPB, $q(\mathrm{~B})$ $=1.51 \mathrm{e}, q\left(\mathrm{~F}^{-}\right)=-0.62$. The charge transfer process increases the solubility of the THFIPB $+\mathrm{F}^{-}$receptor complex in PC. Due to its larger dipole moment in the complexed state $(\mu=2.94$ debye vs $\mu=2.45$ debye), the THFIPB receptor might be more soluble in polar solvents than TPFPB after anion binding. For reference, our calculated value for PC in the gas phase is $\mu=5.46$ debye. Finally, we note that the three fluorine atoms closest to the boron atom that form part of the free-anion receptors form an equilateral triangular cage with a side length around 4.6 A. Upon binding the cage hardly changes in the TPFPB case, while it shrinks to about $4.0 \AA$ in THIFPB. The significance of this for anion capture and release kinetics is currently being investigated.

To help understand the binding and attractive range of THIFPB and TPFPB, we show the highest occupied molecualr orbitals (HOMOs) of both anion receptors in Fig. 8. Notice the more localized nature of the HOMO in THIFPB as compared to the more dispersed HOMO in TPFPB. A more localized orbital provides a more readily available space for the fluoride anion to share its extra electron. Delocalization of the HOMO provides the extra binding energy observed in TPFPB.

The anion receptor must provide a complexation energy that equals or exceeds the sum of its own solvation energy (between -4 and $-10 \mathrm{kcal} / \mathrm{mol}$ for a neutral molecule in PC) plus the solvation energy of $\mathrm{F}^{-}$in PC. We refer to this excess enthalpy as a binding energy. Binding energies at close range were calculated and are shown as negative (exothermic) enthalpies in Fig. 8. Either of these binding energies is high for anion capture, but relatively small when compared to a true covalent bond (typically on the order of $100 \mathrm{Kcal} / \mathrm{mol}$ ). Thus, the binding is reversible and anion release is easily accomplished at the voltages used in the charge cycle (around $5 \mathrm{~V}$ ), which are strong enough to break covalent bonds in the absence of a stable solvent such as PC.

If the binding energy of the anion receptor is too weak LiF will remain in the solid precipitate phase, and if the binding energy is too high the $\mathrm{F}^{-}$will remain bound to the receptor and it will either not be intercalated or the $\mathrm{F}^{-}$and anion receptor will be co-intercalated. The anion receptor is required to provide the right balance for an exchange of $\mathrm{F}^{-}$from the LiF crystal to the intercalated electrode. Fortunately the $\mathrm{F}^{-}$intercalation energy is calculated to be very favorable, ca. -183 and $-88.6 \mathrm{kcal} / \mathrm{mol}$ from vacuum and from pure PC solution, respectively. Other processes include the endothermic electron transfer to graphite, not included in this discussion.

The anion receptor-fluoride binding energy is calculated from the energy of the optimized geometry in PC in the complexed form

Table II. Anion receptor binding energy model parameters.

\begin{tabular}{|c|c|c|c|c|c|c|c|}
\hline & $\begin{array}{c}D o \\
(\mathrm{Kcal} / \mathrm{mol})\end{array}$ & $\begin{array}{l}R o \\
\text { (A) }\end{array}$ & $\zeta$ & $\begin{array}{c}E b \\
(\mathrm{Kcal} / \mathrm{mol})\end{array}$ & $\begin{array}{l}R b \\
\text { (A) }\end{array}$ & $\begin{array}{c}\beta \\
(\mathrm{I} / \mathrm{A} 2)\end{array}$ & $\begin{array}{c}\text { Release Barrier } \\
D o+E b \\
(\mathrm{Kcal} / \mathrm{mol})\end{array}$ \\
\hline THFIPB-F- & 29.4 & 1.439 & 7.553 & 19.7 & 5.839 & 0.068 & 49.1 \\
\hline TPFPB-F- & 39.5 & 1.444 & 6.958 & 19.6 & 5.245 & 0.100 & 59.2 \\
\hline
\end{tabular}




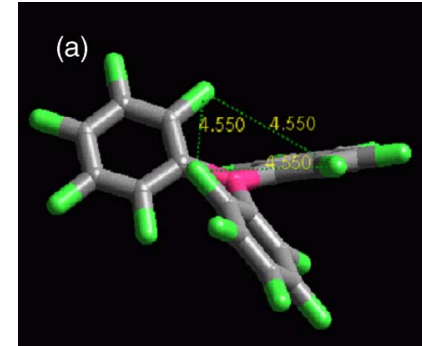

(c)

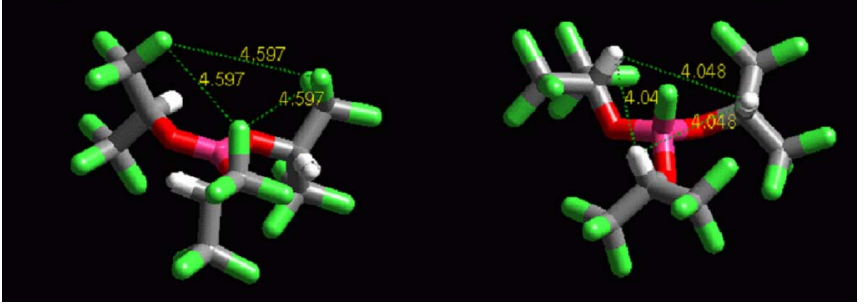

Figure 6. (Color online) (a) Uncomplexed form of TPFPB. The phenyl groups have a propeller-like conformation (angle degrees). (b) In the complexed form the propeller angle, and dipole moment, increase (33 to 73 degrees and 0.27 to 2.45 debye, respectively). (c) The uncomplexed form of THFIPB has all hydrogens on the same plane as the borate (BO3) group. (d) Upon complexation THFIPB the hydrogens point out of the plane, a change of 60 degrees. The dipole moment increases from 0.51 to 2.94 debye. The distances shown are for between the atoms closest to the captured $\mathrm{F}^{-}$anion. In the case of THFIPB these are the three tertiary hydrogens.

compared to the energy of the individual fragments (anion receptor $+\mathrm{F}^{-}$) at infinity. For validation we calculated the binding energy of TPFPB at $10 \AA$. The resulting difference is reasonably close to zero $(-0.84 \mathrm{kcal} / \mathrm{mol})$. Figure 8 shows the binding energy as a function of distance. Figure 7 shows the corresponding molecular geometries as calculated in solution. We determined the basis set superposition error correction for a few receptor/F distances and found these corrections to be small, on the order of $1 \mathrm{kcal} / \mathrm{mol}$.

We fit the binding energies with a model, Eq. 1, which represents the attractive part of the potential as a Morse curve plus a Gaussian repulsive curve

$$
E_{\mathrm{vdw}}=D_{o}\left[e^{-\zeta\left[\left(R / R_{o}\right)-1\right]}-2 e^{-(\zeta / 2)\left[\left(R / R_{o}\right)-1\right]}\right]+E_{b} e^{-\beta\left(R-R_{b}\right) 2}
$$

Here, $D_{o}$ and $R_{o}$ are the total binding energy and receptor- $\mathrm{F}^{-}$equilibrium distance, $E_{b}$ and $R_{b}$ the energy and position of the desolvation barrier. TPFPB has a total binding energy of $39.5 \mathrm{kcal} / \mathrm{mol}$ and an equilibrium distance of $1.43 \AA$ and THFIPB binds weaker, $29.4 \mathrm{kcal} / \mathrm{mol}$ at $1.44 \AA$ (Fig. 8). The positive energies at around $10 \AA$ for both TPFPB and THFIPB, are the "desolvation" energy barrier of the fluoride ion $\mathrm{F}^{-}$. This effect is due to the limited amount of continuum dielectric medium (PC solvent) to sufficiently solvate the $\mathrm{F}^{-}$charge just before it gets released by the anion receptor. Our model predicts a barrier for capture of around $20 \mathrm{kcal} / \mathrm{mol}$ at a dis-

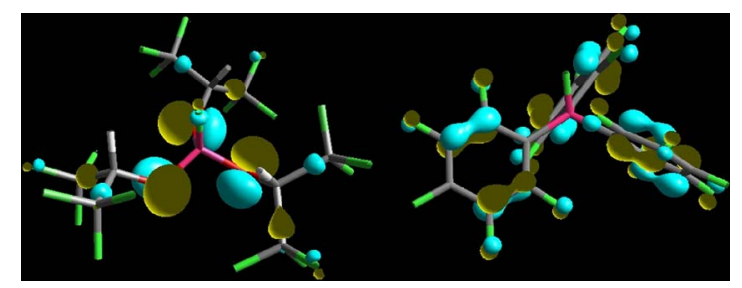

Figure 7. (Color online) Calculated HOMO of THIFPB (left) and TPFPB (right) anion receptors with bound $\mathrm{F}^{-}$anion. The more delocalized HOMO in the phenyl groups of TPFPB corresponds to a more strongly bound fluorine atom.

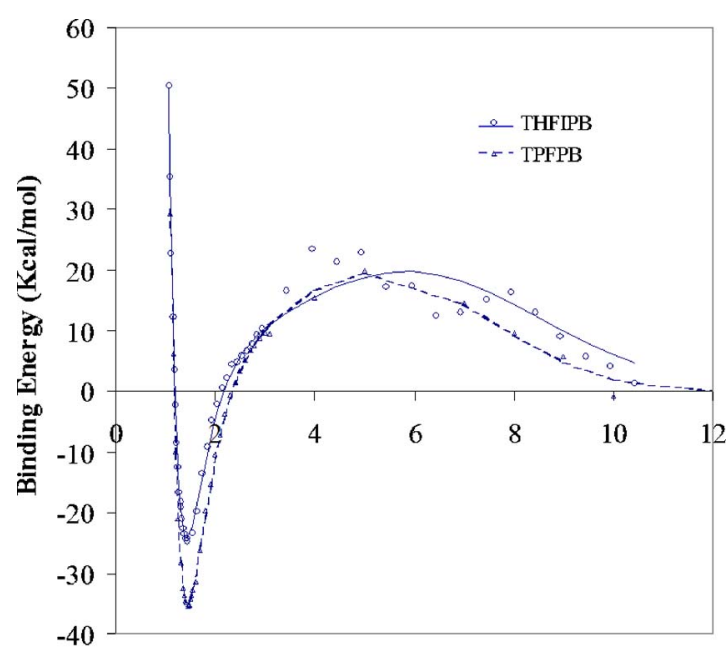

B.....F- Distance (A)

Figure 8. (Color online) Binding energetics of two anion receptors at various fluoride ion distances. Note the longer attractive range of THIFPB (beginning at $3.2 \AA$ ) when compared to TPFPB (2.3 $\AA$ ). The desolvation barrier at a distance of about $5 \AA$ dominates the anion release kinetics. The lines are the fit Morse plus Gaussian barrier given in Eq. 1 and Table II.

tance of about 5-6 $\AA$ for both TPFPB and THFIPB, respectively (Table II). This should have a significant effect on capture and release kinetics at low to moderate temperatures. The barrier to be overcome for anion release is the sum of binding and desolvation barriers. The release barrier values shown in Table II indicate that TPFPB has a significantly higher, by about $10 \mathrm{kcal} / \mathrm{mol}$, anion release barrier than THFIPB. This might explain the experimentally measured superior performance of THFIPB over TPFPB in terms of cathode-specific capacity. We conclude that anion receptor release, which is a kinetically driven process, is responsible for the performance difference between TPFPB and THFIPB, and not the thermodynamics of anion capture. Additional simulations are underway to identify anion receptors with moderate anion binding energies, a much lower anion release barrier, and a suitably wide electrochemical stability window.

\section{Conclusion}

We have demonstrated a route to reversibly intercalate fluorideanion receptor complexes in graphite via a nonaqueous electrochemical process. Key limitations of the cell design have been assessed, including the electrochemical stability window of the electrolyte solution. The practical specific capacity of the intercalated species in graphite is limited to about $60-80 \mathrm{mAh} / \mathrm{g}$, which was likely due to the relatively large size of the fluoride-anion receptor complex. Synchrotron X-ray diffraction, impedance spectroscopy, and ${ }^{11} \mathrm{~B}$ NMR data support the contention that co-intercalation of the anion receptor with fluoride occurs in preference to the desired fluoride intercalation mechanism. Ab initio calculations give preliminary evidence that the thermodynamic relationships of the anion receptor capture of fluoride in the electrolyte are of importance for solubility, but that the barrier to fluoride release may dominate the kinetics of anion release and the ultimate energy density performance.

\section{Acknowledgments}

This work was carried out at the Jet Propulsion Laboratory, California Institute of Technology, under contract with the National Aeronautics and Space Administration, funded through the JPL Research and Technology Development fund. The authors thank S. Surampudi for providing guidance throughout the project and Adri van Duin for valuable comments on the theoretical section. Portions of this research were carried out at the Stanford Synchrotron Radia- 
tion Laboratory, a national user facility operated by Stanford University on behalf of the U.S. Department of Energy, Office of Basic Energy Sciences.

California Institute of Technology assisted in meeting the publication costs of this article.

\section{References}

1. K. Guerin, J. P. Pinhheiro, M. Dubois, Z. Fawal, F. Masin, R. Yazami, and A. Hamwi, Chem. Mater, 16, 1786 (2004).

2. H. Takenaka, M. Kawaguchi, M. Lerner, and N. Bartlett, J. Chem. Soc., Chem. Commun., 19, 1431 (1987).

3. M. Noel, R. Santhanam, and M. F. Flora, J. Power Sources, 56, 125 (1995).

4. Y. Sato, T. Kume, R. Hagiwara, and Y. Ito, Carbon, 41, 351 (2003).

5. A. M. Panich, T. Nakajima, and S. D. Goren, Chem. Phys. Lett., 271, 381 (1997).

6. T. Ishihara, M. Koga, H. Matsumoto, and M. Yoshio, Electrochem. Solid-State Lett., 10, A74 (2007).

7. F. P. McCullough and A. F. Beale, U.S. Pat. 4,865,931 (1989).

8. R. T. Carlin, H. C. De Long, J. Fuller, and P. C. Trulove, J. Electrochem. Soc., 141, L73 (1994).

9. J. A. Seel and J. R. Dahn, J. Electrochem. Soc., 147, 892 (2000).

10. J. R. Dahn and J. A. Seel, J. Electrochem. Soc., 147, 899 (2000).

11. See, for example, H. S. Lee, X. Q. Yang, C. L. Xiang, and J. McBreen, J. Electrochem. Soc., 145, 2813 (1998).
12. J. McBreen, H. S. Lee, X. Q. Yang, and X. Sun, J. Power Sources, 89, 163 (2000).

13. X. Sun, H. S. Lee, X. Q. Yang, and J. McBreen, J. Electrochem. Soc., 149, A355 (2002).

14. K. Xu and C. A. Angell, J. Electrochem. Soc., 149, A920 (2002).

15. T. P. Onak, H. Landesman, R. E. Williams, and I. Shapiro, J. Phys. Chem., 63, $1533(2000)$.

16. Y. Matsuo, M. Segawa, J. Mitani, and Y. Sugie, J. Fluorine Chem., 87, 145 (1998).

17. W. Yan and M. M. Lerner, J. Electrochem. Soc., 148, D83 (2001); W. Yan and M. M. Lerner, J. Electrochem. Soc., 150, D169 (2003); W. Yan and M. M. Lerner, J. Electrochem. Soc., 151, J14 (2004); W. Yan, L. Kabalnova, N. Sukpirom, S. Zhang, and M. M. Lerner, J. Fluorine Chem., 125, 1703 (2004).

18. A. D. Becke, J. Chem. Phys., 98, 5648 (1993).

19. C. Lee, W. Yang, and R. G. Parr, Phys. Rev. B, 37, 785 (1998).

20. Jaguar, version 6.5, Schrodinger, LLC, New York, NY (2005).

21. P. J. Hay and W. R. Wadt, J. Chem. Phys., 82, 299 (1985).

22. D. J. Tannor, B. Marten, R. Murphy, R. A. Friesner, D. Sitkoff, A. Nicholls, B. Honig, M. Ringnalda, and W. A. Goddard, J. Am. Chem. Soc., 116, 11875 (1994).

23. Y. H. Jang, M. Blanco, S. Dasgupta, D. A. Keire, J. E. Shively, and W. A. GoddardIII, J. Am. Chem. Soc., 121, 6142 (1999).

24. NIST Standard Reference Databases Number 101, R. D. Johnson III, Editor, NIST (2005).

25. Handbook of Chemistry and Physics, D. R. Lide, Editor, pp. 10-211, CRC Press, Boca Raton, FL (1992).

26. C. Blondel, C. Delsart, and F. Goldfarb, J. Phys. B, 34, L281 (2001) 